\section{Greenwashing durch klimasmarte Landwirtschaft}

\author{
Der Klimawandel trifft die Landwirtschaft in Entwicklungsländern \\ besonders hart. Dürren und Überflutungen gefährden die Nah- \\ rungsmittelproduktion und die Einkommensgrundlagen von \\ kleinbäuerlichen Produzenten. Agrarkonzerne versuchen, davon \\ zu profitieren - und machen eifrig Werbung für eine klima- \\ smarte Landwirtschaft. Von Marita Wiggerthale
}

W ir sind von der Dürre schwer betroffen. Alle unsere Tiere sind verendet. Wir hatten 50 Ziegen“, erzählt die 50-jährige Lule Abrahn aus Äthiopien. Mehr als 60 Millionen Menschen sind vom Mega-El Nino 2015/2016 betroffen gewesen und litten vielfach unter Hunger. Er vermittelt eine Ahnung von den katastrophalen Folgen des Klimawandels für die Menschen, die von der Landwirtschaft leben. Drängende Fragen müssen beantwortet werden: Welche Form des Ökosystemmanagements macht die Landwirtschaft lokal widerstandsfähiger? Wie können die Risiken für besonders gefährdete und marginalisierte Gruppen reduziert werden?

\section{Guter Lösungsansatz oder Trojanisches Pferd?}

Einige Regierungen, internationale Organisationen und Agrarkonzerne behaupten, mit der klimasmarten Landwirtschaft (Climate-Smart Agriculture, kurz CSA) bereits eine Antwort darauf gefunden zu haben. Doch CSA ist weder smart, noch wirklich gut für das Klima, warnen zivilgesellschaftliche Organisationen wie Oxfam. Sie halten CSA für ein ausgeklügeltes Greenwashing-Projekt und ein gefährliches trojanisches Pferd: Statt die Hungernden und vom Klimawandel betroffenen Menschen zu unterstützen, würde CSA vor allem die auf massiven Pestizideinsatz basierende, sogenannte Grüne Revolution fortführen, die industrielle Agrarproduktion zementieren und bestehende Machtungleichge- wichte im Welternährungssystem verfestigen.

Bereits ein oberflächlicher Blick auf die im Jahr 2014 gegründete Global Alliance for Climate-Smart Agriculture belegt, wie berechtigt diese Bedenken sind. Denn die Allianz definiert überhaupt nicht, was klimasmart ist. Mit anderen Worten: CSA stellt einen Freibrief für jegliche umweltschädliche Anbaupraktiken dar und erlaubt Umweltsündern die globale Klimaagenda für sich zu vereinnahmen. Schließlich können selbst der massive Einsatz von Glyphosat und die Verwendung von gentechnisch veränderten Organismen als klimasmart gelten. Dazu passt, dass die Mitglieder der Global Alliance for Climate-Smart Agriculture hauptsächlich Industrieländer und multinationale Konzerne bzw. ihre Interessenverbände sind. Die Wirksamkeit ihres Beitrags müssen sie aber weder belegen, noch darüber berichten. Es gibt also gute Gründe, warum die Bundesregierung an ihrer Position festhalten sollte, nicht der Global Alliance for ClimateSmart Agriculture beizutreten.

\section{Technokratische Lösungen}

Aber auch sonst taugt CSA nicht als Lösungsansatz für die Bewältigung von Klimakrisen. Hunger ist kein Problem des Mangels, sondern der Verteilung. Und auch Armut und die Anfälligkeit gegenüber Klimakrisen haben vor allem soziale Ursachen. CSA ignoriert dies und setzt einseitig auf technische Lösungen, wie die pfluglose Bodenbearbeitung.
Als Anbaupraxis der industriellen Landwirtschaft fördert sie Monokulturen, die mit einem massiven Einsatz von Düngemitteln und hochgiftigen Pestiziden verbunden sind.

Wen wundert es da, dass Chemieriesen wie Monsanto und Syngenta die klimasmarte Landwirtschaft unterstützen. Sie propagieren diese umweltschädliche Praxis als Beitrag zum Klimaschutz, weil durch den Verzicht auf das Pflügen mehr Kohlenstoff im Boden gespeichert wird. CSA schafft aber auch Anreize für Investoren, mit dem Handel von Bodenkohlenstoffzertifikaten Gewinne zu erzielen und dafür Land im großen Stil zu erwerben. Die Folge wäre noch mehr Landgrabbing.

\section{Neue politische Rahmenbedingungen}

Im Rahmen von CSA haben auch die Menschenrechte keine Priorität. Menschen, die von der Landwirtschaft leben und besonders von den Klimakrisen betroffen sind, müssten eigentlich gestärkt und geschützt werden, zum Beispiel, indem man ihnen den Zugang zu Land und Wasser ermöglicht. Auch die Gerechtigkeit zwischen den Geschlechtern sollte gefördert werden, schließlich sind Frauen stärker als Männer vom Klimawandel betroffen.

Wer die Landwirtschaft klimafreundlicher gestalten und besser an den Klimawandel anpassen will, muss unser gesamtes Ernährungssystem ökologisch und sozial nachhaltiger ausrichten. Was wir brauchen, ist eine neue Landwirtschafts- und Handelspolitik, die das Recht auf Nahrung, agrarökologische Prinzipen und Ernährungssouveränität als Leitlinien hat.

AUTORIN + KONTAKT

Marita Wiggerthale ist Agrarexpertin bei Oxfam Deutschland.

Oxfam Deutschland e. V. Am Köllnischen Park 1, 10179 Berlin. E-Mail: mwiggerthale@oxfam.de. Website: www.oxfam.de
No Derivates License (http://creativecommons.org/licenses/by-nc-nd/4.o/ deed.de), which permits copying and redistributing the material in any medium or format, provided the original work is properly cited, it is not used for commercial purposes and it is not remixed, transformed or built upon. 